\title{
Association of BMI, socioeconomic status and menarche age with anemia in rural school going adolescent girls
}

\author{
Kavthekar $S^{1}$, Kulkarni $D^{2}$, Kurane $A^{3}$,Chougule $A^{4}$ \\ ${ }^{1}$ Dr Saiprasad Kavthekar, Assistant Professor, Department of Pediatrics, ${ }^{2}$ Dr Devayani Kulkarni, Assistant Professor, \\ Department of Pediatrics, ${ }^{3} \mathrm{Dr}$ Anil Kurane, Professor and HOD, Department of Pediatrics, ${ }^{4}$ Dr Ashok Chougule, \\ Assistant Professor, Department of Pediatrics. All are affiliated with Dr D.Y. Patil Medical College and Hospital \\ Kadamwadi, Kolhapur, Maharashtra, India.
}

Address for Correspondence: Dr. Saiprasad Kavthekar, Department of Pediatrics, Dr D. Y. Patil Medical College and Hospital Kadamwadi, Kolhapur, Maharashtra, India. Email: saiprasadka@yahoo.co.in

\begin{abstract}
:
Background: India has the world's highest prevalence of anemia among women and adolescent girls. Rural adolescent girls are particularly vulnerable to develop anemia. Aims and Objectives: To study the prevalence of anemia in rural school going adolescent girl sand its associated factors like BMI, socioeconomic status and age at menarche. Materials and Methods: A prospective study was carried out among 1200 adolescent girls (12-16 yrs) of 10 randomly selected government schools. BMI, Socioeconomic status by modified kuppuswami Classification, Menarche age by history and hemoglobin by cyanmethemoglobin method of all adolescent girls were obtained and analysed statistically. Results: Theoverall prevalence of anemia in rural adolescent girls was $54.2 \%$, highest $63.3 \%$ was in the age group of 13 to $14 \mathrm{yrs}$, $30 \%$ suffered from moderate anemia. BMI categorization showed 48.6\%, 42.2\%, 9\% and 1.2\% adolescent girls were normal, underweight, overweight and obese respectively. Underweight adolescent girls were significantly anemic (74.2\%) compared to normal $48.6 \%$, overweight $39.2 \%$ and obese $40 \%$. Class V and IV of socioeconomic status adolescent girlswere significantly anemic $74.7 \%$ and $60 \%$ respectively when compared to Class III (42.6\%), Class II $(42.85 \%)$ and Class I (12\%). The adolescent girls having menarche $\leq 13$ yrs had significant anemia. Conclusion: The prevalence of anemia in rural adolescent girls was high. Under weight, class V and IV socioeconomic status and earlier age at menarche girls were at risk of developing anemia. The governments anemia prevention and control programme should focus on rural adolescent girls.
\end{abstract}

Keywords: Adolescent girls, Anemia, BMI, Menarche, Socioeconomic status

\section{Introduction}

Anemia is defined as a low level of hemoglobin in the blood and it is one of the world's most widespread nutritional problems [1]. Theprevalence of anemia among adolescents is $27 \%$ in developing countries and $6 \%$ in developed countries [2]. India has the world's highest prevalence of iron deficiency anemia among women, with $60-70 \%$ of the adolescent girls being anemic [3]. It has an impact on psychological and physical development, behavioural and work performance [4]. Association of anemiawith adverse maternal outcome such as puerperal sepsis, antepartum haemorrhage and maternal mortality is no longer a

Manuscript received: $2^{\text {nd }}$ June 2016

Reviewed: $12^{\text {th }}$ June 2016

Author Corrected; $25^{\text {th }}$ June 2016

Accepted for Publication: $9^{\text {th }}$ July 2016 debatable subject [5]. Apart from the risk to the mother, it is also responsible for increased incidence of premature births, low birth weight babies and high perinatal mortality [6].

Adolescence is a period of transition between childhood and adulthood occurring between 12-18yrs of age. During adolescence anemia is more prevalent due to growth spurt, onset of menarche, poor nutrition and poverty. Anemia prevalence is usually higher in rural areas than urban areas. This stresses the need to investigate the factors associated with prevalence of anemia. Prevention of anemia is effective when the strategy is focused right from adolescence for their future reproductive life. There are very few studies focussing on anemic adolescent girls [7,8,9,10,11]. 
Thepresent study was designed to study the prevalence of anemia in rural school going adolescent girls and its associated factors like body mass index, socioeconomic status and age at menarche.

\section{Materials and Methods}

This prospective study was conducted in ten government schools of rural Kolhapur, among apparently healthy 1200 adolescent girls. Schools were chosen randomly and from each school 120 adolescent girls and from each Class 30 adolescent girls were selected randomly from attendance register. The girls between the age 12-16yrs were selected for the study. The study was carried out after approval by Institutional ethical committee and permission from school principal/ headmaster. Informed consent from parents/ guardian was also taken.

Body weight was measured with light clothing and without shoes in the upright position to the nearest 100 grams, using calibrated electronic balance. Height was measured without shoes to the nearest $0.1 \mathrm{~cm}$ using calibrated portable stadiometer. Body mass index (BMI) was calculated by weight in $\mathrm{kg}$ divided by height squared in meter square. As per recommendation of Khadilkar etal [12], adolescent girls were categorised according to their BMI using BMI percentile curves for Indian girl from 5 to 18 yrs. They were Classified as: underweight $\left(\mathrm{BMI}<3^{\text {rd }}\right.$ percentile), normal (BMI $3^{\text {rd }}$ percentile to adult equivalent of $\mathrm{BMI}<23$ ), overweight (Adult equivalent of BMI 23 to adult equivalent of 27.99) and obese (adult equivalent of $\mathrm{BMI} \geq 28$ ). Modified kuppuswami socio-economic status was used to divide the study groups into five Classes: Class I (upper), Class II (upper middle), Class III (lower middle), Class IV (upper lower), Class V (lower). [13] This is an important tool in hospital and community based research in India. This scale takes account of education, occupation and income of family to Classify study group in five Classes depending upon score. Taking inflation and price rise this has been modifiedwith conversion factor for the income. All adolescent girls were asked to recall their age at menarche and menstrual history were also obtained. Hemoglobin level was determined by cyanmethemoglobin method (type of colorimetric method). Blood was collected by finger prick, 20 microliter of blood sample was mixed with $5 \mathrm{ml}$ of Drabkin's solution at the spot by micro pipette. Hemoglobin in the blood is converted into cyanomethhemoglobin. The absorbance of cyanomethhemoglobin was measured at $540 \mathrm{~nm}$ by photoelectric colorimeter on the same day of sample collection. As per WHO recommendations anaemia was diagnosed when $\mathrm{Hb}<12 \mathrm{~g} / \mathrm{dL}$ in 10-18 years girls [14]. The severity of anaemia was graded as mild $(\mathrm{Hb}>10 \mathrm{~g} / \mathrm{dL})$, moderate $(\mathrm{Hb} 7-9.9 \mathrm{~g} / \mathrm{dL})$ and severe $(\mathrm{Hb}<7 \mathrm{~g} / \mathrm{dL})$. [15] All observations were recorded, tabulated and subjected to statistical analysis by Chisquaretest, in which $\mathrm{P}<0.05$ was significant. The $\mathrm{P}$ value was determined by using the primer of biostatistics and MS Excel 2007.

\section{Results}

A total 1200 adolescent girls, from 10 schools, 120 girls from each school and 30 girls from each Classrandomly selected were included in this study. The study showed that the overall prevalence of anemia was $54.2 \%$ $(1200 / 650)$ in rural school going adolescent girls. The study results emphasised the high prevalence of anemia among all age groups of adolescent girls.

The highest prevalence is in the age group of 13-14 yrs $(63.3 \%)$ and minimum in the age group of $12-13 y r s$ (46\%) [Table- I] 30\% adolescent girl suffered from moderate anemia while $23.4 \%$ and $0.8 \%$ suffered from mild and severe anemia respectively [Figure I]

Table I: Age wise distribution of anemic adolescent girls. $\left(\mathrm{X}^{2}=21.523\right.$ with 3 degrees of freedom; $\left.\mathrm{P}=0.000\right)$

\begin{tabular}{|c|c|c|c|c|c|c|}
\hline \multirow{2}{*}{ Age in yrs } & \multicolumn{3}{|c|}{ Adolescent girls } \\
\cline { 2 - 7 } & \multicolumn{2}{|c|}{ Anemic } & \multicolumn{2}{c|}{ Non anemic } & \multicolumn{3}{c|}{ Total } \\
\cline { 2 - 7 } & No & $\mathbf{\%}$ & No & No & \% \\
\hline $12-13$ & 138 & 46 & 162 & 54 & 300 & 100 \\
\hline $13-14$ & 190 & 63.3 & 110 & 42.6 & 300 & 100 \\
\hline $14-15$ & 172 & 57.4 & 150 & 50 & 300 & 100 \\
\hline $15-16$ & 150 & 50 & $\mathbf{5 5 0}$ & $\mathbf{4 5 . 8}$ & $\mathbf{1 2 0 0}$ & $\mathbf{1 0 0}$ \\
\hline Total & $\mathbf{6 5 0}$ & $\mathbf{5 4 . 2}$ & $\mathbf{5 5 0}$ & & \\
\hline
\end{tabular}




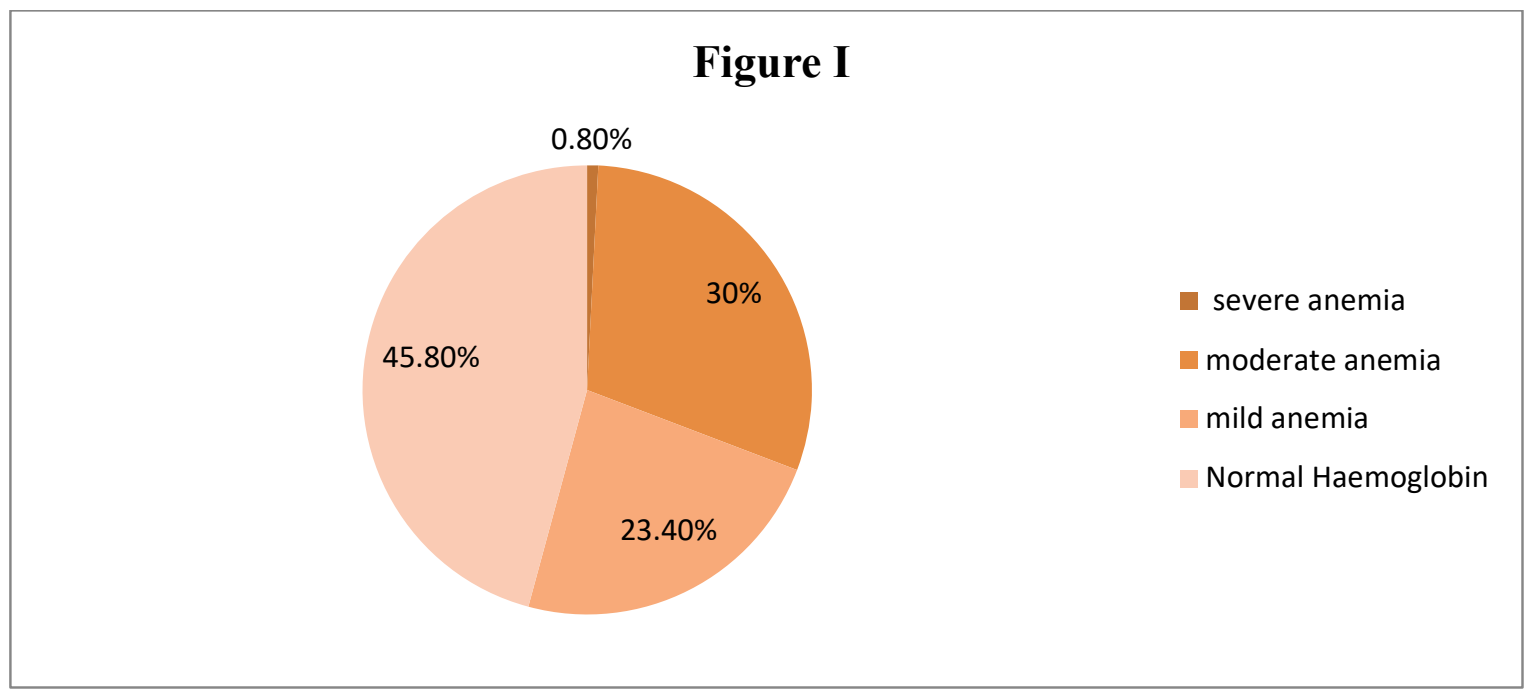

Figure-I: Severity of anemia in adolescent girls.

The study result showed $48.6 \%, 41.2 \%, 9 \%$ and $1.2 \%$ adolescent girls were from normal, underweight, overweight and obese respectively in BMI category. Underweight adolescent girls hadsignificantprevalence of anemia (74.2\%) as compared to normal $48.6 \%$, overweight $39.2 \%$ and obese $40 \%$ [Table II]. When severity of anemia was compared, underweight adolescent girls were significantly had severe (1.4\%) moderate $(45.3 \%)$ and mild $(27.5 \%)$ anemia as compared to normal, overweight and obese adolescent girls. [Figure II]

Table II: Association between body mass index and anemia.

$\left(X^{2}=135.449\right.$ with 3 degrees of freedom; $\left.P=0.000\right)$

\begin{tabular}{|c|c|c|c|c|c|c|c|c|}
\hline \multirow{2}{*}{} & \multicolumn{2}{|c|}{ Normal } & \multicolumn{2}{c|}{ Underweight } & \multicolumn{2}{c|}{ Overweight } & \multicolumn{2}{c|}{ Obese } \\
\cline { 2 - 9 } & No & \% & No & \% & No & \% & No & 40 \\
\hline Anemic & 235 & 40.3 & 367 & 74.2 & 42 & 39.2 & 6 & 60 \\
\hline Non anemic & 348 & 59.7 & 128 & 25.8 & 65 & 60.8 & 9 & 1.2 \\
\hline BMI Total & 583 & 48.6 & 495 & 41.2 & 107 & 9.0 & 15 & \\
\hline
\end{tabular}

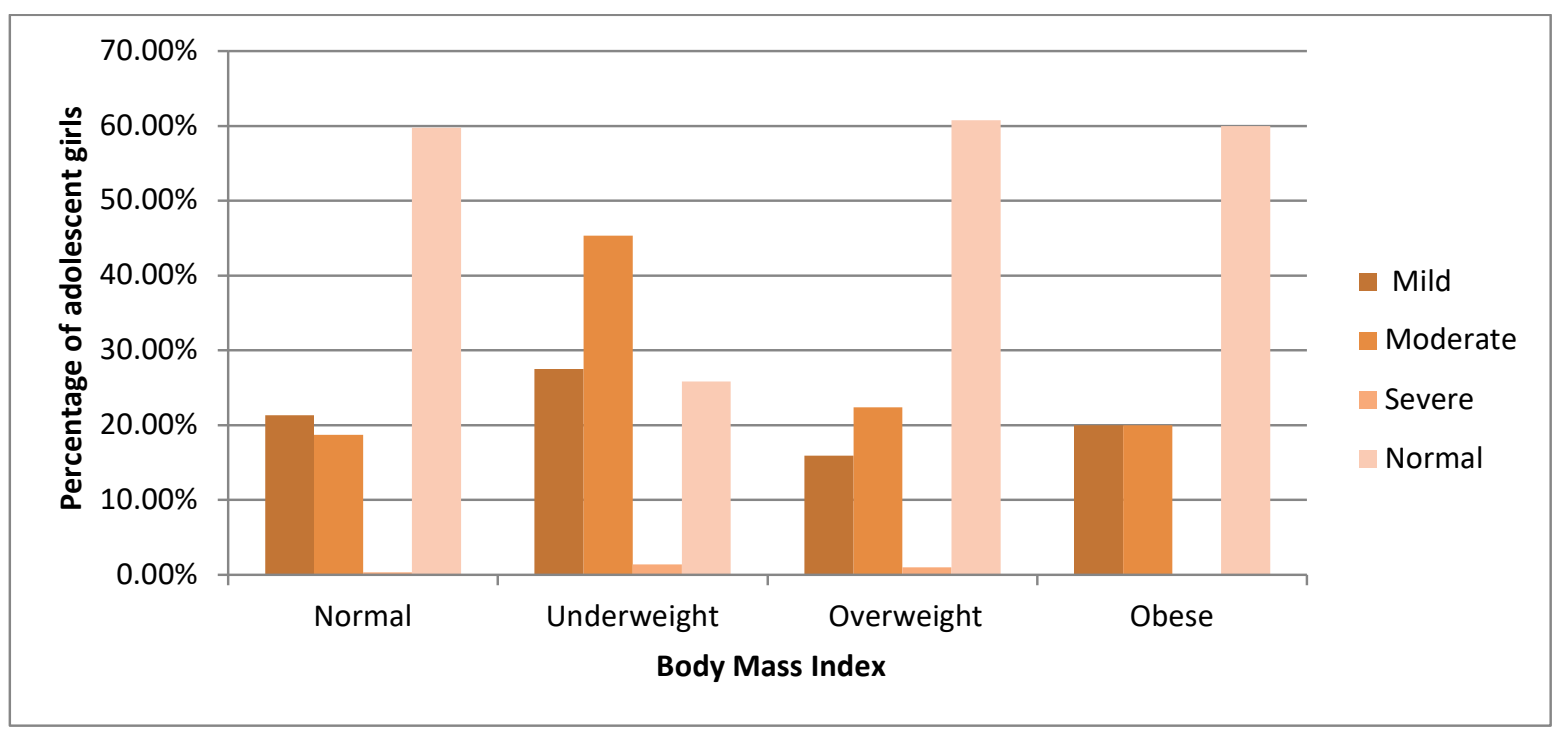

Figure-II: Association between body mass index and severity of anemia $\left(\mathrm{X}^{2}=150.268\right.$ with 9 degrees of freedom; $\left.\mathrm{P}=0.000\right)$ 
Class V (74.7\%) and IV (60\%) of socioeconomic status adolescent girls, were significantly anemicas compared to Class III (42.6\%), Class II (42.8\%) and Class I (12\%) of socioeconomic status. [Table III] Out of socioeconomic Class V adolescent girls suffered significantly severe $(2.6 \%)$, moderate $(56.7 \%)$, and mild $(15.3 \%)$ anemia. Anemia in adolescent girls was significantly reduced with rise in socioeconomic class.

\section{[Figure III]}

Table III: Association between socioeconomic class and anemia in adolescent girls. $\left(\mathrm{x}^{2}=118.696\right.$ with 4 degrees of freedom; $\left.\mathrm{P}=0.000\right)$

\begin{tabular}{|c|c|c|c|c|c|c|c|c|c|c|}
\hline \multirow{2}{*}{} & \multicolumn{8}{|c|}{ Socioeconomic class according to kuppuswami classification . } \\
\cline { 2 - 13 } & \multicolumn{2}{|c|}{ Class I } & \multicolumn{2}{c|}{ Class II } & \multicolumn{2}{c|}{ Class III } & \multicolumn{2}{c|}{ Class IV } & \multicolumn{2}{c|}{ Class V } \\
\cline { 2 - 12 } & No & $\mathbf{\%}$ & No & $\mathbf{\%}$ & No & \% & No & \% & No & \% \\
\hline Anemic & 06 & 12 & 96 & 42.8 & 126 & 42.6 & 198 & 60 & 224 & 74.7 \\
\hline Non anemic & 44 & 88 & 128 & 57.2 & 170 & 57.4 & 132 & 40 & 76 & 25.3 \\
\hline & & & & & & & & & & \\
\hline Total & $\mathbf{5 0}$ & $\mathbf{4 . 2}$ & $\mathbf{2 2 4}$ & $\mathbf{1 8 . 7}$ & $\mathbf{2 9 6}$ & $\mathbf{2 4 . 6}$ & $\mathbf{3 3 0}$ & $\mathbf{2 7 . 5}$ & $\mathbf{3 0 0}$ & $\mathbf{2 5}$ \\
\hline
\end{tabular}

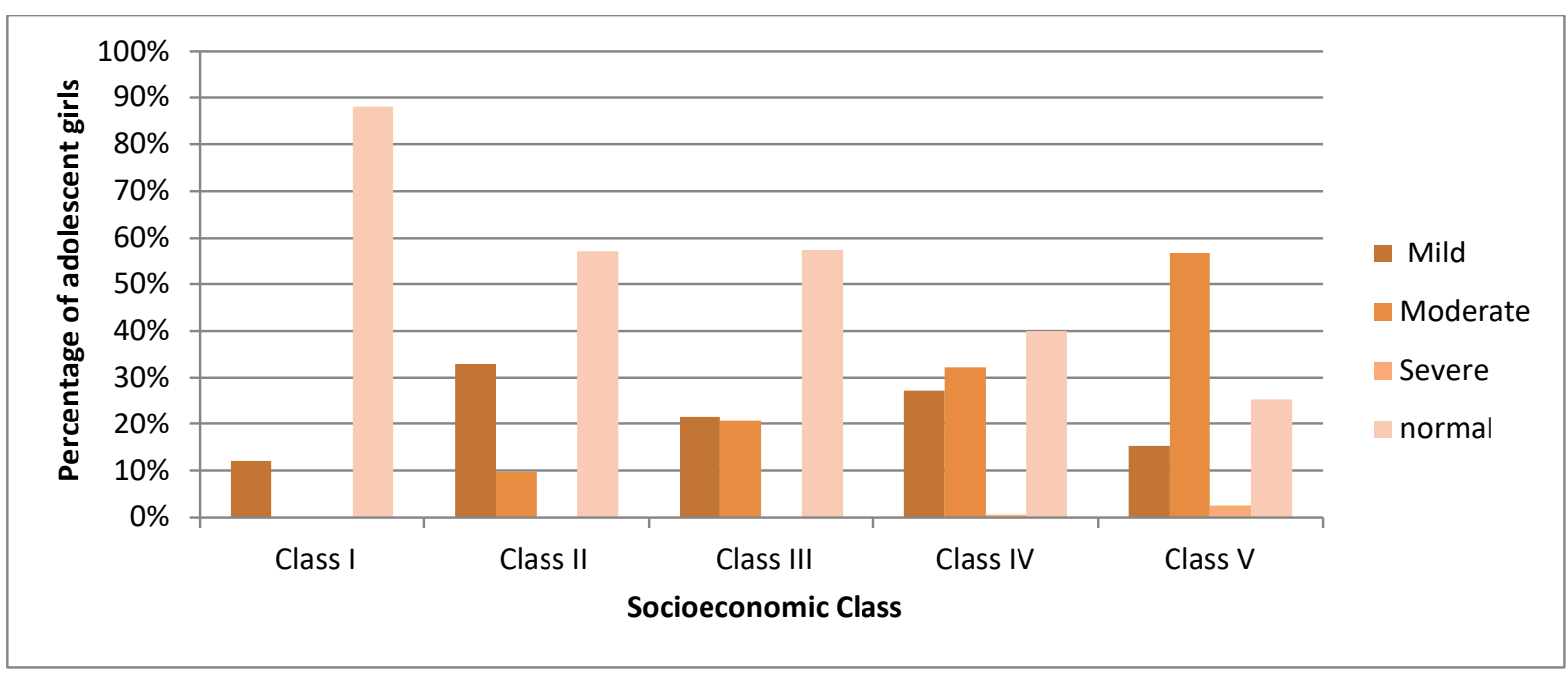

Figure-III: Association between socioeconomic class and severity of anemia $\left(\mathrm{X}^{2}=229.030\right.$ with 12 degrees of freedom; $\left.\mathrm{P}=0.000\right)$

The commonest age at menarche in our study was $12-14$ yrs, with maximum $422(35.2 \%)$ attained at 13 yrs. Thegirls who attained menarche at $\leq 11 \mathrm{yrs}, 12 \mathrm{yrs}$ and $13 \mathrm{yrs}$ had significant anemia $61.3 \%, 68.2 \%$ and $54.3 \%$ respectively. [Table IV] The girls who attained menarche at $\leq 11 \mathrm{yrs}$ of age suffered significantly severe $(8.2 \%)$, moderate $(32.6 \%)$ and mild (20.4\%) anemia. As menarche age increases the prevalence of anemia decreases. [Figure IV]

Table IV: Association between menarche age and anemia in adolescent girls.

$\left(x^{2}=30.753\right.$ with 4 degrees of freedom; $\left.\mathrm{P}=0.000\right)$

\begin{tabular}{|c|c|c|c|c|c|c|}
\hline \multirow[t]{2}{*}{ Menarche Age(yrs) } & \multicolumn{2}{|c|}{ Anemic } & \multicolumn{2}{|c|}{ Non anemic } & \multicolumn{2}{|c|}{ Total } \\
\hline & No & No & $\%$ & $\%$ & No & $\%$ \\
\hline$\leq 11$ & 30 & 49 & 4.0 & 61.3 & 19 & 38.7 \\
\hline 12 & 150 & 220 & 18.4 & 68.2 & 70 & 31.8 \\
\hline 13 & 230 & 422 & 35.2 & 54.5 & 192 & 45.5 \\
\hline 14 & 200 & 410 & 34.1 & 48.8 & 210 & 51.2 \\
\hline $15-16$ & 40 & 99 & 8.3 & 40.4 & 59 & 59.6 \\
\hline Total & 650 & 1200 & 100 & 54.2 & $\mathbf{5 5 0}$ & 45.8 \\
\hline
\end{tabular}




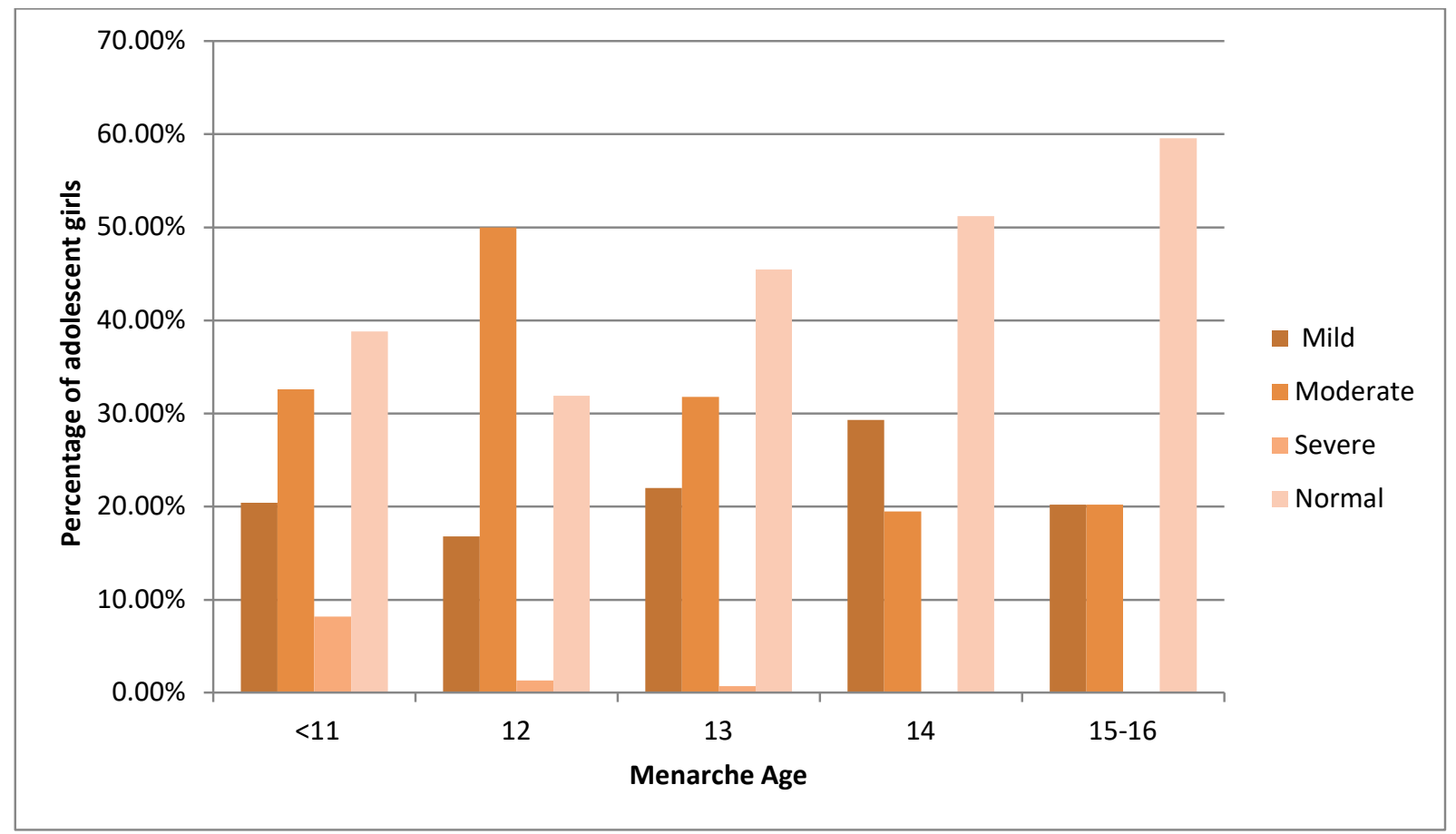

Figure IV: Association between menarche age and severity of anemia. $\left(\mathrm{X}^{2}=112.482\right.$ with 12 degrees of freedom; $\left.\mathrm{P}=0.000\right)$

\section{Discussion}

Anemia symbolizes both poor nutrition and poor health. Adolescence is a crucial phase of growth in the life cycle of an individual. In girls middle adolescence growth happens earlier (12-15yrs) than in boys (13$16 \mathrm{yrs})$. In the present study, prevalence of anemia in rural adolescent girls was reported to be $54.2 \%$. Rawat etal [7], Kaur etal[8] and Kakkar etal [9] reported similar prevalence of anemia as $34.5 \%, 59.8 \%$ and $58.4 \%$ respectively in adolescent girls.

Deshpande etal [11] from Maharashtra, Sudhagandhi etal [16] from Tamil Nadu and Bharti etal [17] from Jharkhand reported prevalence of anemia as $60 \%$, $67.8 \%$ and $99.9 \%$ respectively in adolescent girls. Peter etal [18] and Chitra B etal [19] observed prevalence of anemia as $77.9 \%$ and $81.7 \%$ respectively in rural adolescent girls. Goel S [20] observed low prevalence of anemia $13.3 \%$ in Shimla city.

Kakkar etal [9] reported prevalence of anemia $81 \%$ in early adolescence (10-13yrs) as compared to our study $13-14 y r s(63.3 \%)$. Our study reported moderate anemia $(30 \%)$ in adolescent girls. Similar results were reported by Rawat CMS etal [7], Deshpande etal [11] and Sudhagandhi etal [16] as $40.8 \%, 41.3 \%$ and $34.7 \%$ respectively, except Chitra B etal [19] reported severe anemia $54.36 \%$. In our study underweight adolescent girls were significantly anemic $74.2 \%$. Deshpande etal [11] observed girls falling into thin and severly thin category had high prevalence of anemia. Kaur S [8] and Guptaetal [10] observed BMI did not contribute significantly with anemia. Pinhas-Hamiel O etal [21] found an association between anemia and obesity, due to upregulated hepcidin expression that hampers iron absorption. BMI is the most practical and widely used tool to ascertain an individual'sbody fat mass.

A very high percentage of adolescent girls from low socioeconomic status were found to suffer from anemia in the present study. Rawat etal observed anemia was higher in socioeconomic ClassV (50\%) and it significantly reduced with rise in socioeconomic status. Deshpande etal [11] and. Peter etal [18] observed similar correlation between anemia and socioeconomic class. This may be because of better availability of high quality food with rise in socioeconomic status. Bharti etal [17] did not found significant effect of socioeconomic status alone on anemia.

The commonest age at menarche in our study was $13 \mathrm{yrs}$ $(35.2 \%)$ and there was association between earlier menarche age and risk of anemia. This could be because 
of hormonal changes which occur at the time of onset of menarche and blood loss during menstruation. Vasanthietal [22] found that anemia was higher in postmenarcheal girls than in pre-menarchealgirls of rural area. Kakkaretal [9] observed higher the age at menarche, there are more chances of anemia. Gupta etal [10] found no association between onset of menarche and anemia in adolescent girls.

Iron deficiency anemia and anemia are often used synonymously, as iron deficiency anemia constitutes the major anemia during adolescents. This is due to rapid pubertal growth with sharp increase in lean body mass, blood volume and red cell mass which increases iron requirements for myoglobin in muscles and hemoglobin in the blood. Iron requirement increases two to three folds from a preadolescent level of 0.7 to $0.9 \mathrm{mg}$ iron/day to as much as $1.40-3.27 \mathrm{mg}$ iron / day in adolescent girls [23]. In many developing countries, one half of all children and adolescents fail to achieve their full genetic growth potential due to the combined effects of inadequate nutrition and frequent illness. Moreover due to faulty dietary habits,ignorance and in country like India with multitude of social customs and beliefs cited against women, the prevalence of anemia amongst girls remain quiet high. As per WHO, if the prevalence of anemia is equal to or greater than $40 \%$, it can be considered a severe public health problem otherwise prevalence in the range of $20 \%$ to $39 \%$ is to be considered as moderate one. [24] More effective methods need to be devised for community based hemoglobin testing. Iron supplementation programmes need to include nutrition education programmes. Health education and nutritional advise should be given to the adolescent girls at the time of menarche.

To conclude, prevalence of anemia in rural adolescent girls was $54.2 \%$, with highest $63.3 \%$ in the $13-14 y$ rs age group. $30 \%$ adolescent girls were moderately anemic. Underweight adolescent girls, girls from Class $\mathrm{V}$ andIV socioeconomic status and girls who attained menarche at earlier age were at risk of developing anemia. The governments anemia prevention and control programme should focus on rural adolescent girls.

Contributors: SK, DK: designed the study, collected and analysed the data and drafted the manuscript. AK: supervision of the work and analysed the data. AC: literature search and drafted the manuscript. AK will act as the guarantor. All authors approved the final version of manuscript.
Funding: Nil, Conflict of interest: Nil Permission from IRB: Yes

\section{References}

1. Galloway R. Anemia prevention and control: what works. Part I: Programme guidance. 2003. Population, Health and Nutrition Information Project [PHNI] Jorge Scientific Corporation, Washington D.C

2. Halterman JS, Kaczorowski JM, Aligne CA, Auinger P, Szilagyi PG. Iron deficiency and cognitive achievement among school-aged children and adolescents in the United States. Pediatrics. 2001 Jun; 107(6):1381-86.

3. Biradar SS, Biradar SP, Alatagi AC, Wantamutte AS, Malur PE. Prevalence of anemia among adolescent girls: a one year cross sectional study. Journal of Clinical and Diagnostic Research 2012; 6(3): 372-377.

4. Sandstead HH. Causes of iron and zinc deficiencies and their effects on brain. J Nutr. 2000 Feb;130 (2S Suppl) : 347S-349S.

5. Roy S, Chakravorty PS. Maternal and perinatal outcome in severe anemia. J Obstet GynaeInd 1992; 42: 743-750.

6. Rangnekar AG, Darbari R. Foetal outcome in anemia during pregnancy. J Obstet GynaeInd 1993;43: 172176.

7. Rawat CMS, Garg Sk, Sing JV,Bhatnagar M. Sociodemographic correlates of anemia among adolescent girls in rural areas of district Meerut (U.P). Indian J of community Medicine 2001Oct - Dec; XXVI (4) : 173-175

8. Kaur S, Deshmukh PR, Garg BS. Epidemiological correlates of nutritional anemia in adolescent girls of rural Wardha. Indian J Community Med 2006; 31: 255258

9. Kakkar Rakesh, Kakkar Monica, Kandpal SD, JethaniSumit.Study of anemia in adolescent school girls of Bhopal. Indian $\mathrm{j}$ of Community Health 2011June; 23(1): 38-40.

10. Gupta A, Parashar A, Thakur A, Sharma D. Anemia among adolescent girls in Shimla Hills of north India: does BMI and onset of menarche have a role? Indian J Med Sci. 2012 May - Jun; 66 (5-6) : 126 - 30. doi: 10. 4103 / 0019-5359. 114198. 
11. Deshpande Neelam, Karwa Devkinandan, Agar khedkar Sharad, Deshpande Shishir. Prevalence of anemia in adolescent girls and its correlation with demographic factors. International Journal of medicine and public health 2013 Oct -Dec;3(4):235-239.

12. Khadilkar VV. Khadilkar AV, Borade AB, Chiplonkar SA. Body mass index cut-offs for screening for childhood overweight and obesity in Indian children. Indian Pediatr. 2012 Jan;49(1):29-34. Epub 2011 May 30.

13. Kumar N, Shekhar C, Kumar P, Kundu AS. Kuppuswamy's socioeconomic status scale-updating for 2007. Indian J Pediatr. 2007 Dec;74(12):1131-2.

14. De Mayer EM, Dallman P, Gurney Jm, Hallbarg L, Sood SK, Srikantia SG. Preventing and controlling iron deficiency anemia through primary health care. Geneva: world Health Organisation 1989:P8-9.

15. De Gruchy GC. General principles in the diagnosis and treatment of anemia.In: Penington D, Rush B, Cataldi P, editors. Clinical haematology in medical practice, $4^{\text {th }}$ ed Delhi : CBS Publishers and Distributers; 1986 P59.

16. B Sudhagandhi, Sivapatham Sundaresan, W. Ebenezer William, A Prema. Prevalance of anemia in the school children of Kattankulathur, Tamilnadu, India. Int $\mathbf{J}$ of Nutrition, Pharmacology, Neurological Disease. $2011 ; 1(2): 184-88$.

17. Bharati Premananda, Shome Suparna, Chakrabarty Suman, Bharati Susmita, Pal Manoranjan. Burdan of anemia and its socioeconomic determinants among adolescent girls in India.Food and Nutrition Bulletin. The United Nations University 2009; 30(3):217-226.
18. Peter R, Kumar R, Sangwan L, Pandey S. Prevalance of anemia and its correlation to body mass index: Study among unmarried girls. International Journal of Basic and Applied Medical Sciences. 2012 Sept -Dec; 2 (3):58-62.

19. Chitra B, Nair Nisha, Jesni K Jose, Jesina Begam, Nazar Ali, Balkrishna. Study on prevalence of anemia among school children in a rural community setup. Sch Acad J Pharm. 2014;3(6):423-426.

20. GoelS, Gupta BP. Low prevalence of anemia among adolescents of an urban hilly community. Indian $\mathrm{J}$ community Med. 2007; 1:67-68.

21. Pinhas-Hamiel O, New field RS, Koren I, Agmon A, Lilos P, Phillip M. Greater prevalence of iron deficiency in over weight and obese children and adolescents. Int J Obes Relat Metab Disord. 2003 Mar; 27(3):416-8.

22. Vasanthi G, Pawashe AB, Susie H, Sujatha T, Raman L. Iron nutritional status of adolescent girls from rural area and urban slum. Indian Pediatr. 1994 Feb; 31(2):127-32.

23. World Health Organization. Prevention of iron deficiency anemia in adolescents. A role of weekly iron and folic acid supplementation. Geneva: World Health Organization: 2011.

24. WHO/ UNICEF/ UNU (2001). Iron deficiency anemia: Assessment, prevention and control. A guide for programme managers. Report of WHO/ UNICEF/ UNU consultation, 6-10 December 1993, Geneva. Geneva:WHO.

\section{How to cite this article?}

Kavthekar S, Kulkarni D, Kurane A,Chougule A. Association of BMI, socioeconomic status and menarche age with anemia in rural school going adolescent girls. Int J Pediatr Res.2016;3(7):486-492.doi:10.17511/ijpr.2016.i07.04. 